\title{
Facial Sculpturing by Fat Grafting
}

\author{
Behnam Bohluli, Mehran Aghagoli, Farzin Sarkarat, \\ Mansour Malekzadeh and Nima Moharamnejad
}

Additional information is available at the end of the chapter

http://dx.doi.org/10.5772/54874

\section{Introduction}

Autologous fat grafting is one of the most demanded facial cosmetic procedures. Fat reservoirs are usually available in large amounts in most patients. The procedure of fat grafting may be repeated several times without any considerable complications. Facial tissues readily accept autologous fat without any fear of immune reaction or carcinogenicity. It is a popular technique that may be used in maxillofacial esthetic surgery. This procedure may be done as an isolated procedure or as an adjunct to any facial esthetic operation such as face lifting to enhance the final esthetic outcome. The main drawback of this procedure is possibility of resorption and unpredictable results of the augmentation; however it is generally believed that prognosis of fat grafting is directly related to proper case selection and meticulous surgical technique. This chapter provides an overview of current concepts and key points in fat harvesting, refinement and injection that may potentially lead to long-lasting, predictable results. Common complications are discussed, and effort is made to explain ways to avoid these events and to solve the problems when they happen.

\section{History of fat grafting}

The story of fat grafting started in 1893 when a German surgeon (Adolf Neuber) reported his new technique in operating a depressed scar in the infraorbital region of a young man. He harvested a small piece of subdermal fat from the patients upper arm and inserted it to elevate a depressed scar; surprisingly he also explained his frequent failures in treating larger defects and suggested to reserve fat grafting for defects the size of a bean. This effort was occasionally repeated by some other surgeons. The graft results were extremely controversial till 1983 when suction lipectomy was introduced. This technique provided a safe and conservative method 
for fat harvesting and transfer. At this time a new drawback of fat grafting appeared which was resorption and unpredictable results. Coleman explained structural fat grafting with long lasting results. His concept was mainly a refinement of known technique with a great attention to atraumatic handling of fat cells during harvesting, processing and grafting. [1-6] This concept opened a new era in the field of facial esthetic surgery and found its popularity in a really short time; nowadays fat grafting is well-known technique and studies are underway to turn structural fat grafting to a regenerative procedure using stem cells, platelet derivatives and other additives to fat grafts.

\section{Surgical technique}

Fat grafting may be divided into three dominant steps; firstly fat is extracted from a secondary donor site then it is processed and purified by one of the known techniques to separate vital fat cells from other redundant ingredients and finally it is injected or transferred to the recipient site; each step needs crucial attention and plays a role in success of the surgery.

\subsection{Selection of donor site}

Fat harvesting may be done from the lateral thigh, medial thigh, abdomen, suprapubic area and any other part of the body that shows a considerable amount of fat tissue. Some authors believe that medial knee has the least amount of elastic fibers and will lead to a better quality fat, though this finding is not supported by other clinical studies. It is assumed that all donor sites may provide an acceptable amount of vital fatty tissue and patient compliance, surgeon's preference and donor site contours are the main concerns when selecting a donor site. It is sometimes recommended in massive fat harvestings or in lean patients that bilateral donor sites be used to prevent contour deformities [Fig. 1].

\subsection{Donor site preparation and local anesthesia infiltration}

A small 2-3mm stab incision is made, a small cannula is inserted and 20 to 30cc of local anesthetic(lidocaine with 1/200 000 epinephrine) is dispersed in donor site, after 10 to 15 minutes fat harvesting may be started through the same stab incision [Fig. 2].

\subsection{Fat harvesting}

Historically fat harvesting was performed by an open approach and direct resection of fatty tissues; use of microcannulae in 1981 changed fat harvesting techniques to a simple conservative procedure. Cannulae may be connected to a suction machine, negative pressure of the machine takes fat parcels from donor sites toward a sterile reservoir; some authors use a $10 \mathrm{~mm}$ syringe to induce negative pressure in this technique and the cannula is connected to syringe. By withdrawing the plunger, negative pressure is provided, back and forth hand movement will gather fat into the syringe; it is believed that vigorous negative pressure will endanger vital fatty cells and it is proposed that the process of fat harvesting should be based on curettage 


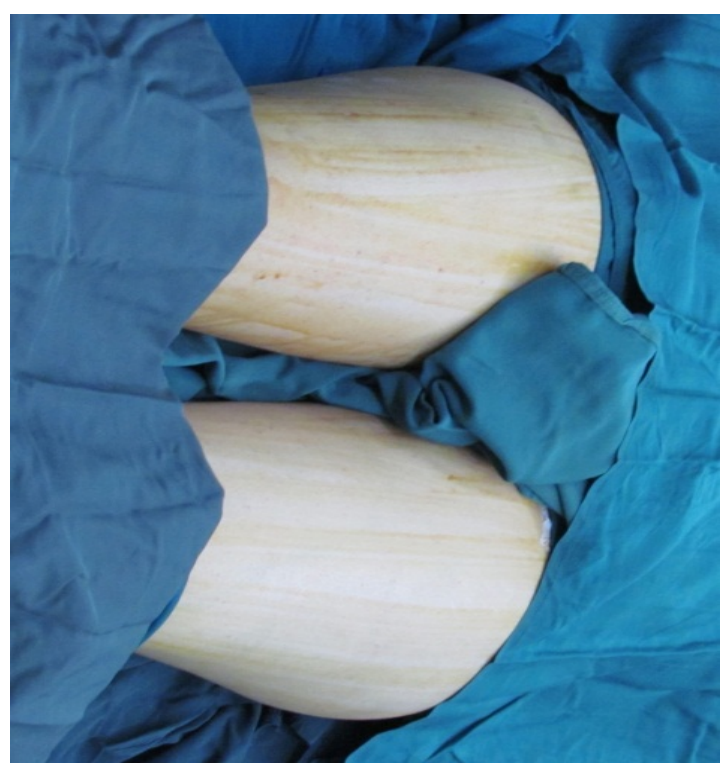

Figure 1. In thin patients bilateral multiple donor sites should be considered; in this patient bilateral medial, lateral thighs and medial knees are prepared.

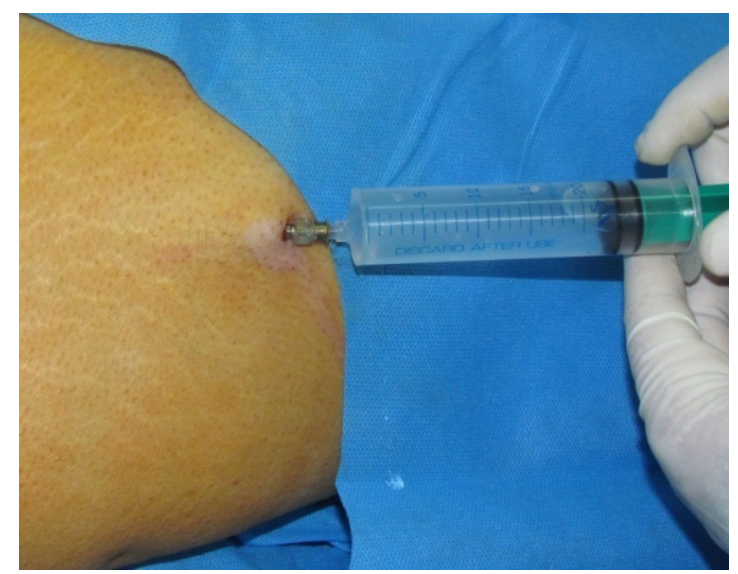

Figure 2. Local anesthetic solution is dispersed into the donor site; it may be done using a 1.5 to $2 \mathrm{~mm}$ cannula.

of several openings located on the lateral sides of a cannula. Slight negative pressure on the plunger of a syringe ( $1 \mathrm{~mm}$ to $3 \mathrm{~mm}$ negative pressure in a 10cc syringe) which is connected to a cannula plus gentle back and forth hand movements in a relatively longer period of suctioning will gather considerable fat in the syringe (Fig. 3).[7-10] 


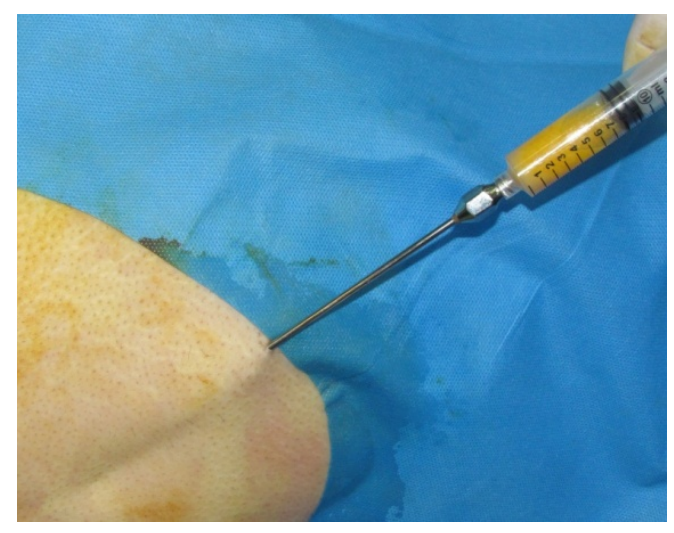

Figure 3. The plunger of a $10 c c$ syringe is withdrawn up to the $3 c c$ mark to induce a light negative pressure.

After 10 to 15 minutes a blunt tip cannula is inserted again and with gentle back and forth movements of the dominant hand fat is extracted from donor adipose tissue while the nondominant hand holds and stabilizes the donor tissue (Fig. 4).

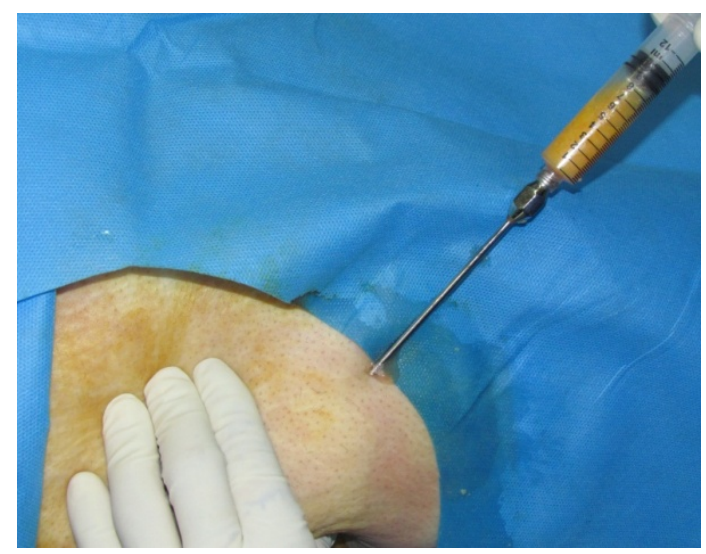

Figure 4. Non-dominat hand holds and stabilizes donor site while dominant hand starts the harvesting procedure.

\subsection{Perils and pitfalls}

1. Vital fat cells are very sensitive, so strict attention to sterility and infection control principles is mandatory; any contamination may lead to infection or destroy the vital cells and result in early resorption.

2. Small diameter cannulae $(2-3 \mathrm{~mm})$ will easily transfer the fat particles and will impose minimal trauma to cells; larger cannulae will accelerate the procedure but take larger 
particles which is not desirable for facial tissues and may potentially deform the donor sites.

3. Low negative pressure (1-3mm negative pressure by withdrawing the plunger up to $3 \mathrm{~mm}$ mark) will take longer but is less traumatic to vital fat cells.

4. Fluid injection. It is usually recommended to infiltrate 1cc of local anesthetics for each cc of harvested fat ; larger quantities of local anesthetics may be added to ringer's solutions. Super wet environment (injection of tumescent solution) which is routinely used in liposuction operations will cause the fat cells to float and may potentially rupture the cells and should be avoided.

5. Blood in harvested fat. It is believed that blood leads to easier and faster degradation of viable fat cells so it is recommended to stop the harvesting process when blood is seen in the harvesting syringe and to proceed to some other donor site to obtain fat.

\subsection{Fat processing}

A usual harvest is a mixture of three main components; the first part is local anesthetics and ringers solution; this part is the solution which is usually injected preoperatively; this liquid is partly transferred to harvested fat and must be separated to eliminate devastating effects of epinephrine on fat cells; the second part is an oily liquid which lacks vital fat cells this liquid has no adverse effect on donor site when injected but it disturbs intraoperative judgments as it increases postoperative swelling and lengthens recovery time so it is best separated from the third and main part which is vital fatty cells. Fat processing includes any procedure that may help to separate fat cells from two other redundant components. Many methods have been introduced for fat processing but the main two are: 1-Centrifuge, 2-filtering and washing.

Centrifuge: harvested fat is poured in 10cc syringes (Fig. 5 a,b).

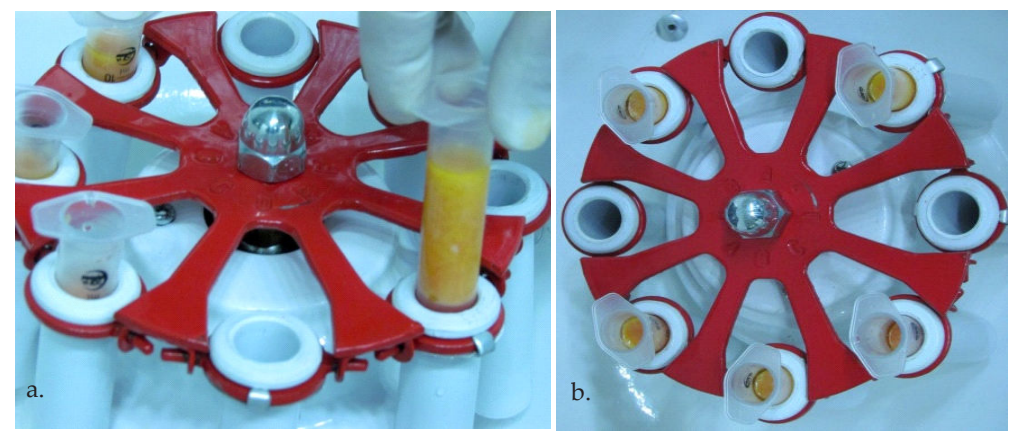

Figure 5. a. Harvested fat is poured in $10 \mathrm{cc}$ syringes. b. Then the syringes are placed in their special slots in the centrifuge machine.

The syringes are inserted in their special slots in a centrifuge and spun at $3000 \mathrm{rpm}$ for 3 minutes to separate different components (Fig. 6 a,b). 

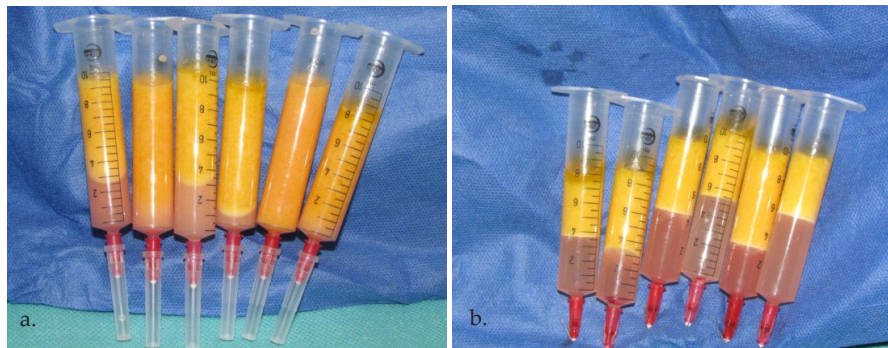

Figure 6. a-Harvested fat is a mixture of lysed fat, local anesthetics and vital fat cells. b-The same view after centrifuge shows the lower part which is local anesthetics and preoperatively injected solutions, middle part is viable fatty tissue and the third upper part is lysed fat cells and triglyceride.

The first part is a liquid that is easily discarded by gentle pressure over the plunger; the second part includes fat cells that are transferred to several 1 cc syringes and are made ready for injection (Fig. 7 a,b,c,d).
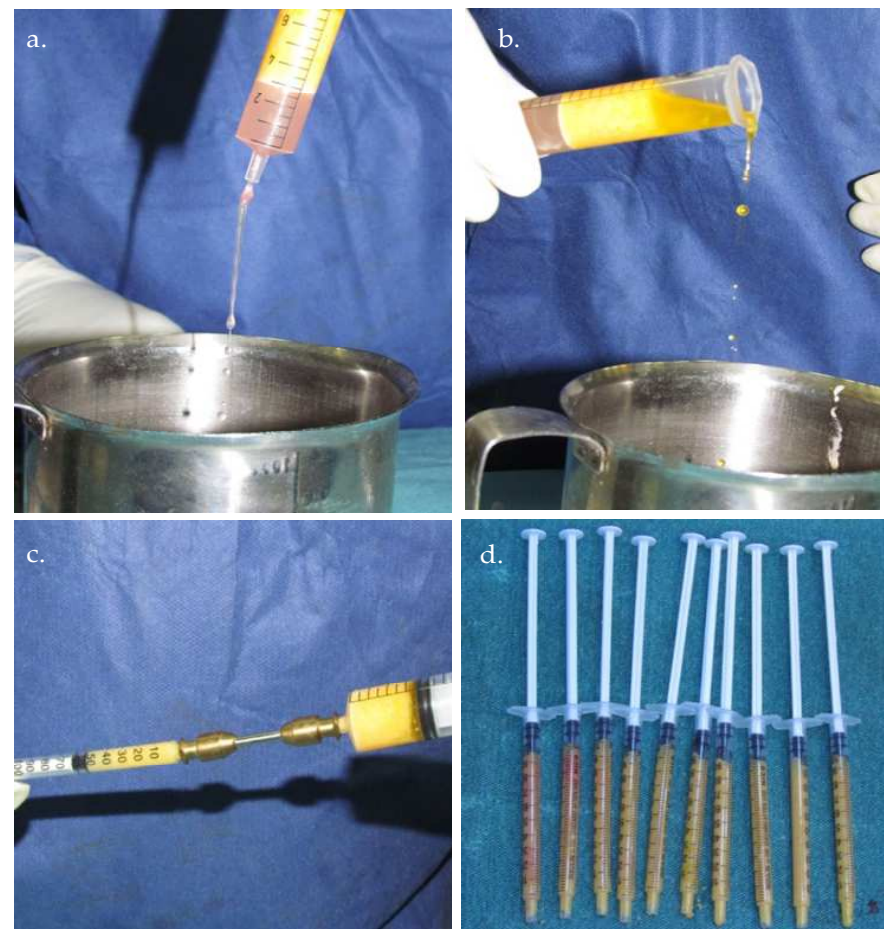

Figure 7. a. In all centrifuged syringes the middle part which is viable fat should be separated by slight pressure over plunger until the first part (local anesthetic) is depleted. b.By gradual turning of syringes the upper part which is lysed fat is easily separated and discarded. c. The middle part which is the main part is transferred to $1 c c$ syringes. d. $1 c c$ syringes are set and ready for injection to recipient sites. 


\subsubsection{Washing and filtering}

Harvested fat is poured in a strainer and washed several times with normal saline; some surgeons close both sides of a strainer and stir it for few minutes to provide a more concentrated fatty compartment. Then one side of the strainer is opened and fat is transferred to 1 cc syringes by a sterile surgical spoon or spatula to get it ready for lipoinjection (Fig 8 a,b, c).
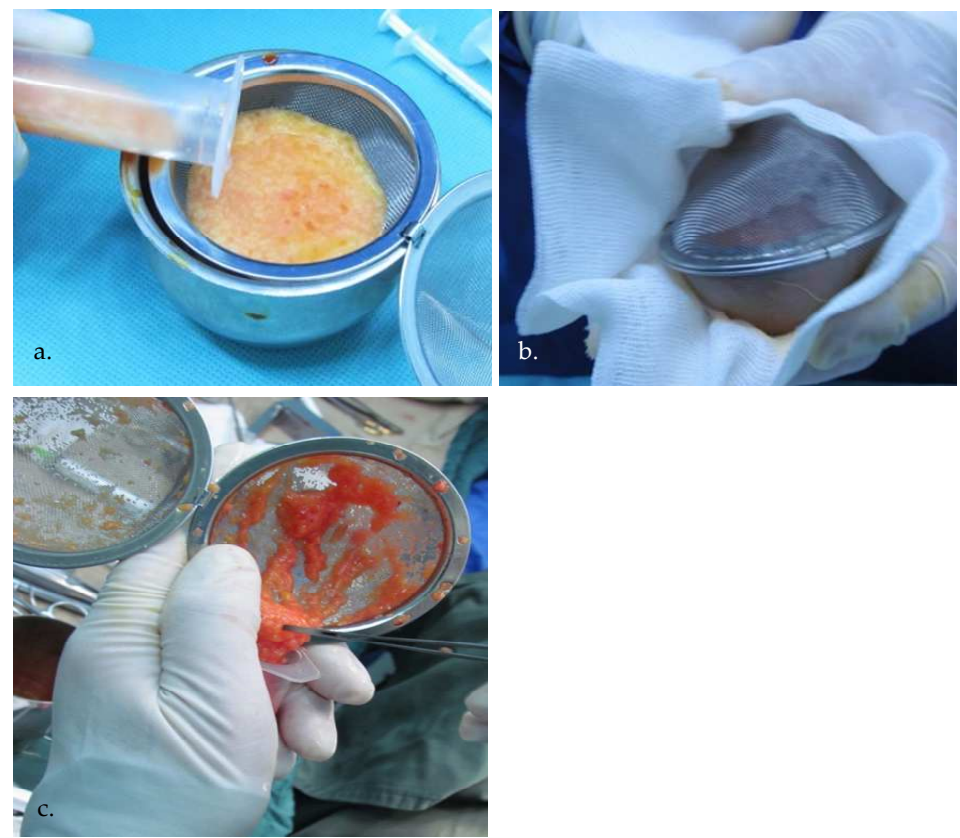

Figure 8. a. Harvested fat is gently poured in a strainer; b. it is washed several times to separate redundant materials.c. Sterile instrument is used to transfer the purified fat to $1 \mathrm{cc}$ syringes.

Selecting processing techniques: Many studies have tried to compare known techniques, up to now none of these trials have convinced the surgeons to leave one technique and unanimously accept the other; but it is clear that skills and expertise, gentle handling of fat and sterility may directly affect the success rate of each technique.

\section{Fat transfer or injection}

Injection sites are carefully designed and marked preoperatively; possible pathways of injection cannulae are drawn with a marker then the usual preparation and draping is performed(Fig. 9 a,b). 

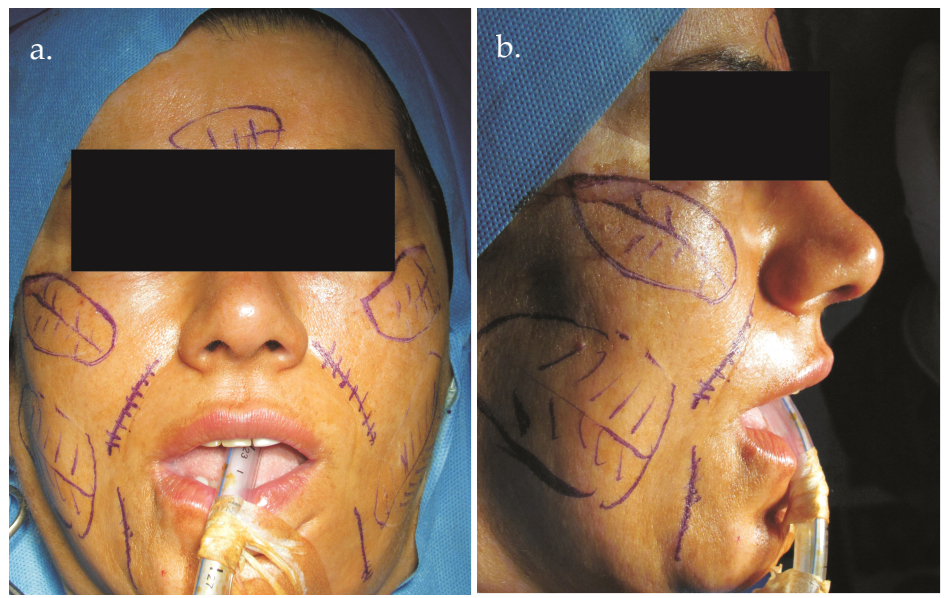

Figure 9. Careful pre-operative drawing and mapping will prevent many post-operative complications.

Proper diameter of injection cannula, amount of graft in each recipient site and injection technique may directly affect the graft viability; these determinant factors will be discussed in detail.

\subsection{Injection technique}

A stab incision is made in pre-planned site cannula is gently inserted.By gentle movement of cannula a tunnel is formed; a small amount of fat $(0.3$ to $0.5 \mathrm{cc})$ is injected while withdrawing the cannula; the process is repeated several times till the total amount of pre-planned fat is delivered to recipient site. A 40 to 60 percent overcorrection may be done to overcome any possible delayed resorption and relapse.

\subsection{Key-points}

\section{Size or diameter of cannula}

The size of the cannula will definitely determine the size of transferred fat particles; these sizes are usually from delicate $0.7 \mathrm{~mm}$ cannulae which are used to fill tear troughs to larger ones (up to $1.5 \mathrm{~mm}$ ) may be used in cheek and chin augmentations.

\section{Regional approaches}

The lips: Lips are mobile and extremely sensitive elements that are challenging sites for augmentation; some authors believe mobility will lead to early resorption while others show long-term stability in their cases. To augment the lips a stab incision is made in center of the lip; left and right sides are separately penetrated by a delicate cannula and $0.5 \mathrm{cc}$ of fat is placed in each side then $0.5 \mathrm{cc}$ is separately inserted in the middle portion. 
Tear troughs: Thin skin with very delicate underling tissue makes this region a critical area in fat grafting; use of a delicate cannula, incremental fat placements in small drops or parcels and meticulous technique of injection may guarantee an acceptable result in periorbital rejuvenation.

The cheek and chin: Malar pads sag with aging; this may lead to flattening of malar contours. This unpleasant deformity may easily be camouflaged by fat grafting; 4cc of fat may be enough to recontour the cheeks. These sites are the most common sites treated. A relatively large (1.2$1.5 \mathrm{~mm}$ ) cannula is usually used to augment chin and cheeks. Chin and cheek augmentation will moderately improve soft tissue contours and should not be accounted as an alternative to hard tissue augmentations (genioplasty, chin implants, malar prosthesis).

Paranasal creases: Elimination of a deep paranasal crease is a big challenge in facial rejuvenation. Filling of nasolabial folds by fat grafting may be added to any face lift procedure or may be performed as a sole procedure; $2-3 c c$ of fat in each site will improve deep nasolabial grooves.

Jaw lines: Gradual appearance of jaw lines and deepening of marionettes line are frustrating sequels of aging; these sites may be easily accessed by small stab incisions that are made for paranasal crease or a separate small incision may made in mandibular border to approach these areas.

Sharp needle injections: Sharp needle injection is a controversial modification of original fat grafting. In this procedure fat is injected transdermally; the main indication of this procedure is to fill deep skin creases or scars.

Amount of injection: The amount of graft may be determined by specific case characteristics though it is generally recommended to use known guidelines and do small modifications from case to case.

\section{Indications for fat grafting}

Fat grafting has been used for many different purposes but it can be generally mentioned that fat graft rehydrates facial skin and improves the patients skin quality; it is also a good filler which may be used to fill a defect, to correct a contour and finally to augment facial volume. Thus, the main indications of fat graft are based on these two dominant properties of fat grafts.

\subsection{Rejuvenation and soft tissue augmentation}

Aging is a complex phenomena it is recently proved that volume loss is one of the main factors that manifests characteristics of an aging face; so fat grafts may potentially restore volume deficits. This procedure may be done solely or added to other rejuvenation procedures such as face or brow lifting [Fig. 10]. 

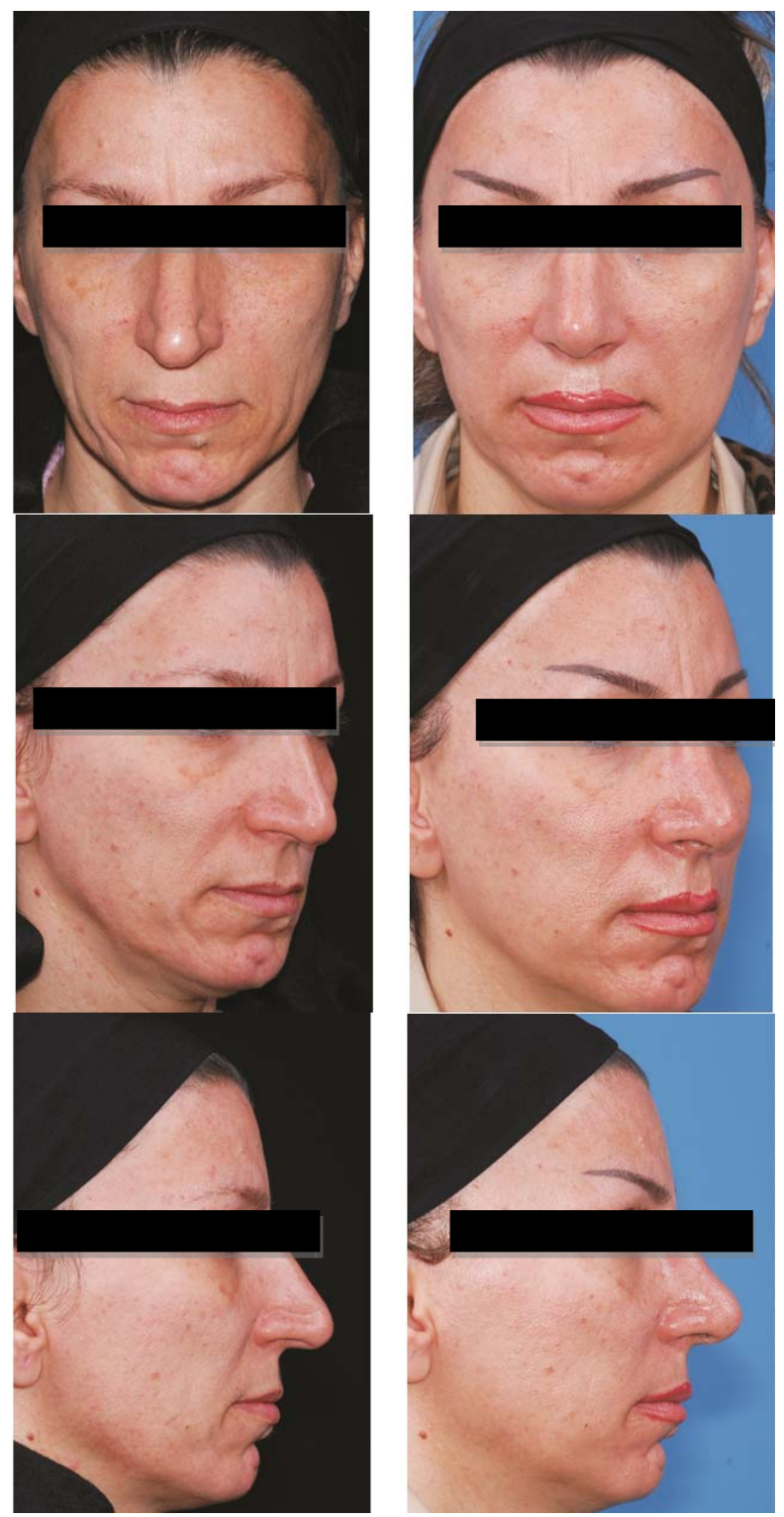

Figure 10. This 41 year-old woman severe characteristics of early aging such as volume loss, deepening of facial creases and loss of skin quality is seen; esthetic nasal surgery and conservative rejuvenation by fat graft was performed. The 1 -year follow-up shows acceptable rejuvenation and improvement of skin quality.

As an adjunct to other major maxillofacial procedure such as rhinoplasty and orthognathic surgery: the role of soft tissue in overall esthetic appearance of the face cannot be underesti- 
mated; fat injection may improve soft tissue conditions and will help the patient to obtain a more pleasant appearance (Fig.11).
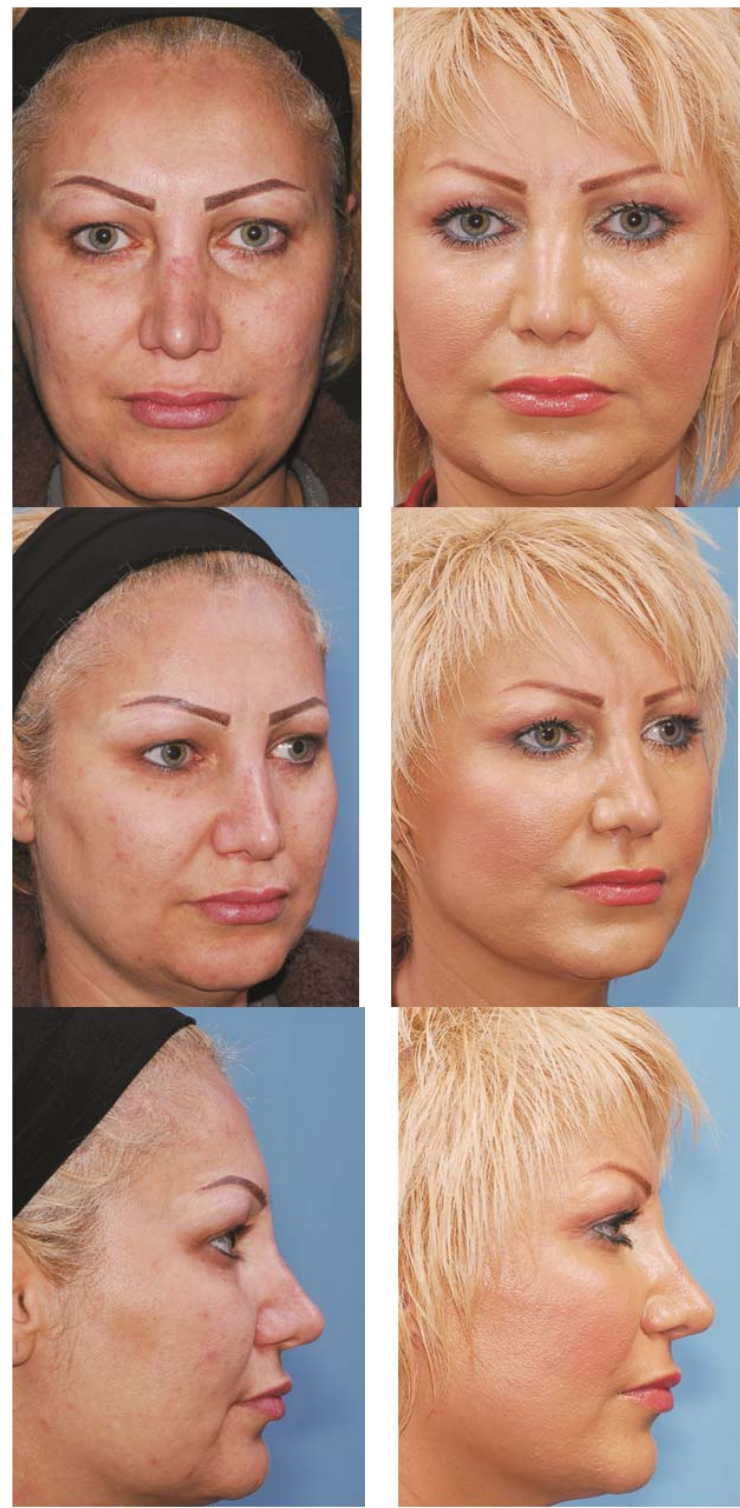

Figure 11. This 53 year-old woman has undergone a minimal-scar face lifting, and the nasolabial folds, malar eminences, nose deformities and marionette lines were simultaneously augmented by fat grafting; the 2-year follow up shows stable results. 


\section{Recontouring facial borders in facial atrophies and hypertrophies}

Fat contouring may be used in camouflaging facial contours which is extremely difficult to correct by other reconstructive modalities. Progressive hemifacial atrophy (Pary-Romberg syndrome),hemifacial hyperplasia, traumatic and developmental facial asymmetries are frequently treated by fat grafting techniques [Fig.12].

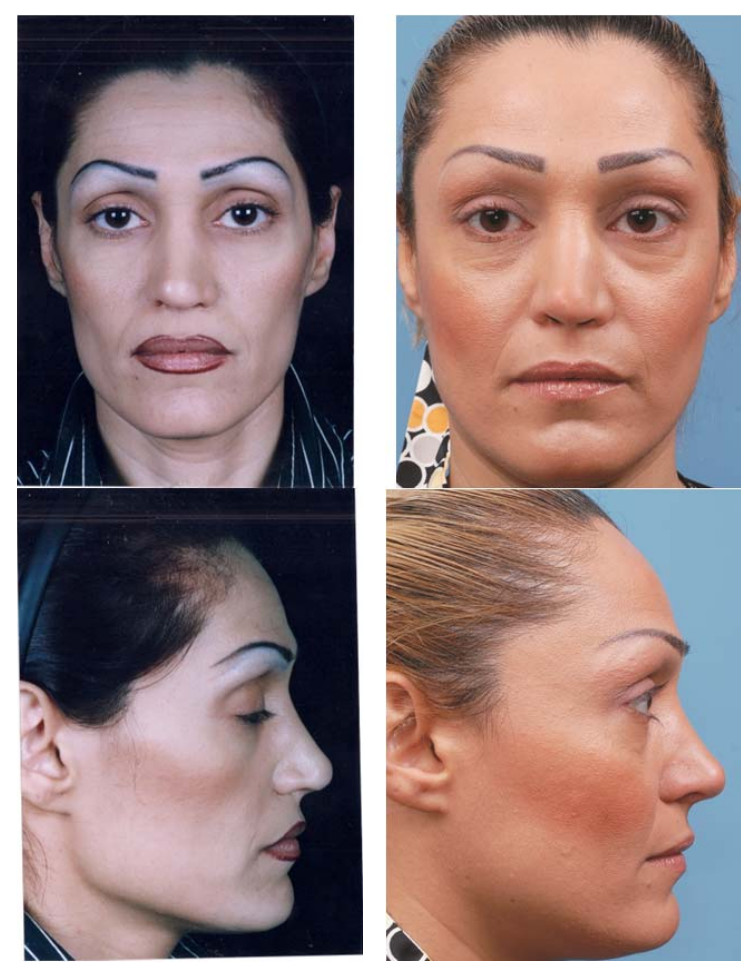

Figure 12. In this 44 year-old woman a masculine face with exaggerated border and contours was planned for feminization; simultaneous forehead lifting, mandibular angle reduction and total facial fat graft was performed. The 10year follow up shows acceptable long term results.

To augment and fill lips, paranasal tissues and cheeks, there is a common trend toward the use of fillers to shape and augment facial tissues; infection, foreign body reactions and carcinogenicity of some fillers has made the fat graft an ideal material. As a filler it may be easily provided in larger amounts, it is cheaper when used in larger amounts and easily accepted by most patients(Fig13).

\section{Fat injection to the nose}

Fat grafting in rhinoplasty is rapidly finding great popularity. Dorsal irregularities after rhinoplasty are extremely challenging in revision rhinoplasty; use of crushed or morselized cartilages or use of a delicate rasping is not usually efficient and sometimes exaggerate the 

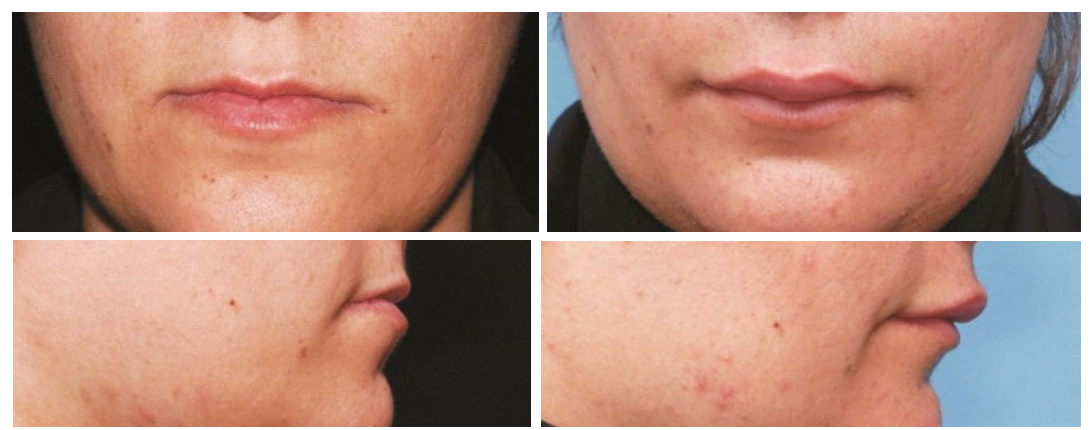

Figure 13. This young class III woman underwent mandibular setback to correct the skeletal deformity. Lack of vermilion show was a frustrating complaint. A 3-year follow up shows long term effects of fat grafts of the upper lip.

problem. Fat injection was recently reported to be effective in these cases; some recent studies advocate the use of fat graft in some primary cases, fat may be used in radix augmentation, dorsal refinements and alar pinch deformities though this field is open to future studies. This harmless but extremely unpredictable technique may be best used in patients with other clear indications of fat grafting as an ancillary procedure in hope to obtain the desired results. $[10,11]$

\section{Complications}

Fat grafting is a relatively safe procedure it is usually followed by some swelling, bruising and ecchymosis both at donor site and facial recipient site; these sequelae are self limiting and will subside spontaneously in maximum two or three weeks.

1. Accumulation of fat particles and visible lumps under the skin: Sometimes small irregularities and lumps are easily seen and palpated under thin skins this will lead to an unesthetic appearance. This complication like most other complications may be best prevented by preoperative planning and delicate surgical technique use of small cannulae in harvesting fat to obtain smaller fat parcels; fat injection and transfer should be done by smaller canulas to help the surgeon delicately place the fat graft in recipient tissues in thin skin areas like lower eyelids and tear troughs; injection may be done in deeper layers.

2. Resorption and relapse: resorption of grafted fat is commonly reported; some authors believe in these cases the procedure should be repeated several times though some studies report long lasting results after one stage surgery; it is unanimously accepted that surgeons skills and expertise directly affects the predictability of results. Sometimes it is suggested to do 40 to 50 percent over-contouring to see the best results after usual estimated resorption.

3. Facial asymmetry: Asymmetries may be due to uneven injections; this complication may be best prevented by proper planning and preoperative mapping over the face; in case 
this asymmetry remains after six months a secondary revision fat grafting may be scheduled.

Immediate postoperative asymmetries in case of precise surgical procedures may be due to asymmetric edema common in facial surgeries and is usually expected to be corrected after subsiding edema.

4. Fat emboli: Fat may be placed in medium to large vessels; these particles may be transferred to vital organs and lead to severe life-threatening problems. Blindness and respiratory dysfunctions are amongst the reported cases. Use of blunt cannulae instead of sharp needles that were previously used for fat injection has considerably reduced this possibility. [12-18]

\section{Donor site complications:}

Surface depressions and contour irregularities: Careless fat resection from a limited area and massive harvesting from a single site may disturb surface integrity of the donor site and may also lead to body asymmetries; it is recommended to harvest the fat in a radial fashion from insertion site to include a wider donor surface. Massive fat resection may be done from two bilateral sites; in case the problems remain after several months it may be restored by a separate fat transfer to damaged donor tissue asymmetric limbs. The total amount of fat which is usually needed in facial fat augmentation will not cause limb asymmetries in normal patients; in thin patients or those who have undergone extensive liposuction procedures both sides should be prepared and a bilateral symmetrical harvest be considered to prevent this unwanted effect. Any possible congenital or developmental preoperative asymmetry should be determined preoperatively and use of the larger limb in asymmetric limbs may help prevent exaggerated limb asymmetries. [19-23]

\section{Author details}

Behnam Bohluli ${ }^{1}$, Mehran Aghagoli ${ }^{2}$, Farzin Sarkarat ${ }^{3}$, Mansour Malekzadeh ${ }^{4}$ and Nima Moharamnejad ${ }^{5}$

1 Craniomaxillofacial Research Center and Department of Oral and Maxillofacial Surgery, Buali Hospital, Islamic Azad University of Medical Sciences, Tehran, Iran

2 Oral and Maxillofacial Surgeon, Private Practice, Tehran, Iran

3 Department of Oral and Maxillofacial Surgery, Buali Hospital, Islamic Azad University of Medical Sciences, Tehran, Iran

4 Craniomaxillofacial Research Center, Islamic Azad University of Medical Sciences,Tehran, Iran

5 Department of Oral and Maxillofacial Surgery, Gazi University, Ankara, Turkey 


\section{References}

[1] Coleman, S. R. Facial augmentation with structural fat grafting.Clin Plast Surg. (2006). Oct;33

[2] Coleman, S. R. Long-term survival of fat transplants: controlled demonstrations.Aesthetic Plast Surg. (1995). Sep-Oct

[3] Coleman, S. R. Facial recontouring with lipostructure.Clin Plast Surg. (1997). Apr

[4] Coleman, S. R. Structural fat grafting.Aesthet Surg J. (1998). Sep-Oct;

[5] Coleman, S. R. Structural fat grafting: more than a permanent filler. Plast Reconstr Surg. (2006). Sep;118

[6] Coleman, S. R. Facial augmentation with structural fat grafting.Clin Plast Surg. (2006). Oct

[7] Grimaldi, M, Gentile, P, Labardi, L, Silvi, E, Trimarco, A, \& Cervelli, V. Lipostructure technique in Romberg syndrome. J Craniofac Surg. (2008). Jul;19(4):

[8] Foyatier, J. L, Mojallal, A, Voulliaume, D, \& Comparin, J. P. Clinical evaluation of structural fat tissue graft (Lipostructure) in volumetric facial restoration with facelift. About 100 cases.Ann Chir Plast Esthet. (2004). Oct;49

[9] Defatta, R. J, \& Williams, E. F. rd.Fat transfer in conjunction with facial rejuvenation procedures.Facial Plast Surg Clin North Am. (2008). Nov

[10] Alencar, J. C, Andrade, S. H, Pessoa, S. G, \& Dias, I. S. Autologous fat transplantation for the treatment of progressive hemifacial atrophy (ParryRomberg syndrome: case report and review of medical literatute). An Bras Dermatol. (2011). Jul-Aug;86(

[11] Hunstad, J. P, Shifrin, D. A, \& Kortesis, B. G. Successful treatment of ParryRomberg syndrome with autologous fat grafting: year follow-up and review. Ann Plast Surg. (2011). Oct, 14.

[12] Teimourian, B. Blindness following fat injections. Plast Reconstr Surg. (1988). Aug

[13] Dreizen, N. G, \& Framm, L. Sudden unilateral visual loss after autologous fat injection into the glabellar area.Am J Ophthalmol. (1989). Jan

[14] Egido, J. A, Arroyo, R, Marcos, A, \& Jiménez-alfaro, I. Middle cerebral artery embolism and unilateral visual loss after autologous fat injection into the glabellar area. Stroke. (1993). Apr

[15] Danesh-meyer, H. V, Savino, P. J, \& Sergott, R. C. Case reports and small case series: ocular and cerebral ischemia following facial injection of autologous fat.Arch Ophthalmol. (2001). May 
[16] Coleman, S. R. Avoidance of arterial occlusion from injection of soft tissue fillers. Aesthet Surg J. (2002). Nov;, 22(6), 555-7.

[17] Smith, P. Adams WP Jr, Lipschitz AH, Chau B, Sorokin E, Rohrich RJ, Brown SA. Autologous human fat grafting: effect of harvesting and preparation techniques on adipocyte graft survival.Plast Reconstr Surg. (2006). May;, 117(6), 1836-44.

[18] Khater, R, Atanassova, P, Anastassov, Y, Pellerin, P, \& Martinot-duquennoy, V. Clinical and experimental study of autologous fat grafting after processing by centrifugation and serum lavage.Aesthetic Plast Surg. 2009 Jan;Epub (2008). Nov 20., 33(1), 37-43.

[19] Monreal, J. Fat grafting to the nose: personal experience with 36 patients. Aesthetic Plast Surg. (2011). Oct; 35(5), 916-22.

[20] Cárdenas, J. C, \& Carvajal, J. Refinement of rhinoplasty with lipoinjection. Aesthetic Plast Surg. (2007). Sep-Oct; 31(5), 501-5.

[21] Teimourian, B, \& Fisher, J. B. Suction curettage to remove excess fat for body contouring. Plast Reconstr Surg. (1981). Jul;, 68(1), 50-8.

[22] Teimourian, B. Repair of soft-tissue contour deficit by means of semiliquid fat graft. Plast Reconstr Surg. (1986). Jul;, 78(1), 123-4.

[23] Illouz, Y. G. The fat cell "graft": a new technique to fill depressions.Plast Reconstr Surg. (1986). Jul;, 78(1), 122-3. 